\title{
Modelling of electrostatic fluidized bed (EFB) coating process using artificial neural networks
}

\author{
M. Barletta*, A. Gisario, S. Guarino \\ Dipartimento di Ingegneria Meccanica, Università degli Studi di Roma "Tor Vergata”, Via del Politecnico, 1-00133 Roma (I), Italy
}

Received 9 January 2006; received in revised form 7 June 2006; accepted 23 June 2006

Available online 1 September 2006

\begin{abstract}
This paper involves experimentation on coating process of metal substrates in an electrostatic fluidized bed (EFB). Several operational parameters were covered like coating time, applied voltage and gas flow rate fed to the fluidized bed.

First, a design of experiment (DOE) approach was used to define the experimental campaign and a general linear model based on analysis of variance (ANOVA) was used to elaborate and interpret the influence of all the operational parameters on coating thickness trends.

Second, the experimental data were modelled using artificial neural networks. Different neural networks and training algorithms were employed to find the best technique to predict the coating thickness trends. The reliability of the best neural network solutions was checked by comparing them with a built ad hoc regression model. The multi-layer perceptron (MLP) neural network trained with backpropagation (BP) algorithm was found to be the fittest model. Besides, a genetic algorithm (GA) was also employed to improve the capability of MLP model to provide the best fit of experimental results all over the investigated ranges.

Finally, a verification experimental plan was performed and a related analytical model was developed to check the reliability of the neural network model with GA to predict the whole coating thickness trends according to the operational parameters. A comparison between the neural network model and an analytical model was also carried out.
\end{abstract}

(C) 2006 Elsevier Ltd. All rights reserved.

Keywords: Electrostatic fluidized bed; Coating process; Neural networks

\section{Introduction}

Over the last two decades, painting and coatings industry has undergone significant changes in chemicals of raw materials (Weiss, 1997). New products like waterborne, high solid contents, UV curable and powder paints have seen their market share remarkably increased. In particular, the market share of powder coatings has been characterized by a noteworthy evolution. In 1994, about $6 \%$ of the whole painting market belonged to the powder paints (Weiss, 1997). In 1999, this share turned to $9 \%$ (Anon, 1995). In 2005, a further increase to $15 \%$ of the whole market has been predicted (Cudin, 2005). The increase in the powder paints diffusion is attributed to four essential factors: the good performance and quality

\footnotetext{
${ }^{*}$ Corresponding author. Tel.: + 390672597168; fax: + 39062021351.

E-mail address: barletta@mail.mec.uniroma2.it (M. Barletta).
}

exhibited by the formulation of newest products, which assure fair aesthetic aspects and good properties of resistance to corrosion, wear and durability; the progressive reduction of products cost in spite of an increase in overall costs of raw organic materials; the low energy consumption connected to the curing process because of the dryness of the powder coating; the low environmental impact and contamination connected to the massive use of products in powder, being the residuals absolutely dry and the products solvent free.

The fast evolution of painting products was not associated to analogous changes in application systems. In particular, powder paints are always applied by electrostatic spray deposition, while electrostatic fluidized bed (EFB) and conventional hot dipping fluidized bed are used just for special applications such as the coating of metal coils, tubes, bands, chains and wires (ASM Metal Handbook, 1995) as well as the coating of peculiar 
components like rotors, fences, webs, appliances, external furniture and so on (ASM Metal Handbook, 1995). Besides, the running and control of coating processes as well as the settings of operational parameters were entrusted to the experience of skilled technicians (Guskov, 2002), to the politics of small adjustments (Barletta and Tagliaferri, 2006a) and, at best, to the employment of empirical models (Barletta and Tagliaferri, 2006b). Nevertheless, process modelling and optimization are very important issues in coating engineering and coating processes are too complicated to be empirically managed (Guskov, 2002), to justify the use of simple analytical models often based on many assumptions, which contradict reality (Barletta et al., 2006) or to have recourse to complicated finite elements (FE) (Barletta et al.) or volume elements (VE) models (Ali and Inculet, 2000; Ye et al., 2002), which cannot be implemented on line and often require laborious calibration procedures and heavy and recursive solution methods (Ye and Domnick, 2003).

Because of the complexity of coating processes, neural network models, which can map the input/output relationships, possess massive parallel computing capability and which can be implemented 'real time' (Cho, 2001), represent a valid alternative to conventional modelling solutions. Besides, several industrial segments in manufacturing already employ neural network models for control and automation purposes (Cho, 2001). On the contrary, in the authors' knowledge, no significant contributions about the employment of neural network solutions in modelling and simulation of coating process can be found in the technical and scientific literature.

In this context, our paper focuses its attention on an application of neural network solutions to an EFB coating process of metal thin sheets with hybrid epoxy-polyester powder paints. First, a design of experiment (DOE) approach was used to investigate the operational parameters of EFB coating process, that is, coating time, applied voltage and air flow fed to the fluidized bed. In such a way, the trends of coating thickness according to process parameters were also achieved. An approach based on the analysis of variance (ANOVA) was also used to assess the meaningfulness of each operational parameter and to evaluate the simple and interaction effects. Second, a wide set of neural network models was examined to determine the best solution in predicting experimental trends and in simulating the whole coating process. Multilayer perceptron (MLP), general feed forward multi-layer perceptron (GFF-MLP) and radial basis function (RBF) neural networks models employing standard back-propagation (BP) and Levenberg-Marquardt (LM) algorithms were trained, tested and their performances were comparatively evaluated. MLP trained with BP algorithm displayed the best performance. Genetic algorithms (GAs) to train the best MLP model were also tested and it was found to be the finest solution to improve the simulation process and to increase the accuracy in coating process modelling. However, a validation of both the MLP model with BP algorithm and GA was performed comparing their capabilities in predicting experimental results with the capability of a built ad hoc regression model.

Finally, a verification experimental plan was performed to check the reliability of the MLP model with GA to predict the experimental trends of coating thickness according to the operational parameters and a straight comparison between the related analytical model and the best neural network solution was also proposed.

\section{Experimental apparatus and procedure}

\subsection{Fluidized bed system and materials}

A thorough description of the EFB system and of the deposition process is reported in a previous paper (Barletta and Tagliaferri, 2006b). For the purposes of the present work, it is important to remark that our fluidization system consisted of a circular grounded column $250 \mathrm{~mm}$ in diameter and $500 \mathrm{~mm}$ in height. A porous plate distributor in polymeric material $8 \mathrm{~mm}$ thick and a homogenization section $250 \mathrm{~mm}$ in diameter and $200 \mathrm{~mm}$ in height were employed (Fig. 1). A fixed bed of hybrid epoxy-polyester powders (dielectric constant of about 3 and surface resistivity around $10^{11}-10^{12} \mathrm{~W} /$ square) $230 \mathrm{~mm}$ in height was pre-loaded in the column onto the porous plate distributor. The powder had a distribution peak set at $25 \mu \mathrm{m}$ with $90 \%$ of the distribution below $55 \mu \mathrm{m}$. With such powders, the fluidized bed had a minimum fluidization flow of $0.65 \mathrm{~m}^{3} / \mathrm{h}$ corresponding to a minimum fluidization velocity of $0.0032 \mathrm{~m} / \mathrm{s}$. The bubbling regime was maintained within an airflow range of $3.5-7 \mathrm{~m}^{3} / \mathrm{h}$ corresponding to superficial velocities 5-10 times the minimum fluidization velocity. Bubbling regime was still on at a flow rate of $10-11 \mathrm{~m}^{3} / \mathrm{h}$, but powder spouts started to rise from dense phase of the bed to the free board, hence discouraging the employment of faster airflows. A set of electrodes fed by a high voltage supply $(\mathrm{dc} 0-100 \mathrm{kV})$, with a negative polarity on electrodes, was placed inside the porous plate distributor of the fluidized bed so that the powder material would be charged as the fluidizing air, coming from homogenization section, caused it to rise up.

From low carbon steel thin sheets, $2 \mathrm{~m}$ long, $1 \mathrm{~m}$ wide and $1 \mathrm{~mm}$ thick, workpieces $100 \mathrm{~mm}$ long and $50 \mathrm{~mm}$ wide were cut by fine blanking. The employed metal substrate was characterized by a very low resistivity (nearly $10 \mathrm{~m} \Omega \mathrm{cm}$ ) suitable for electrostatic deposition. Before being coated, the workpieces were polished by abrasive blast cleaning, followed by phosphoric acid cleaning, and then a water rinse, a chromic acid rinse, and a forced-air rinse. A primer was applied all over the surface of the metal substrate to make it ready for EFB coating process.

\subsection{Experimental procedure and plans}

After paint pre-treatments, primer application and dimensional controls, workpieces were electrically 


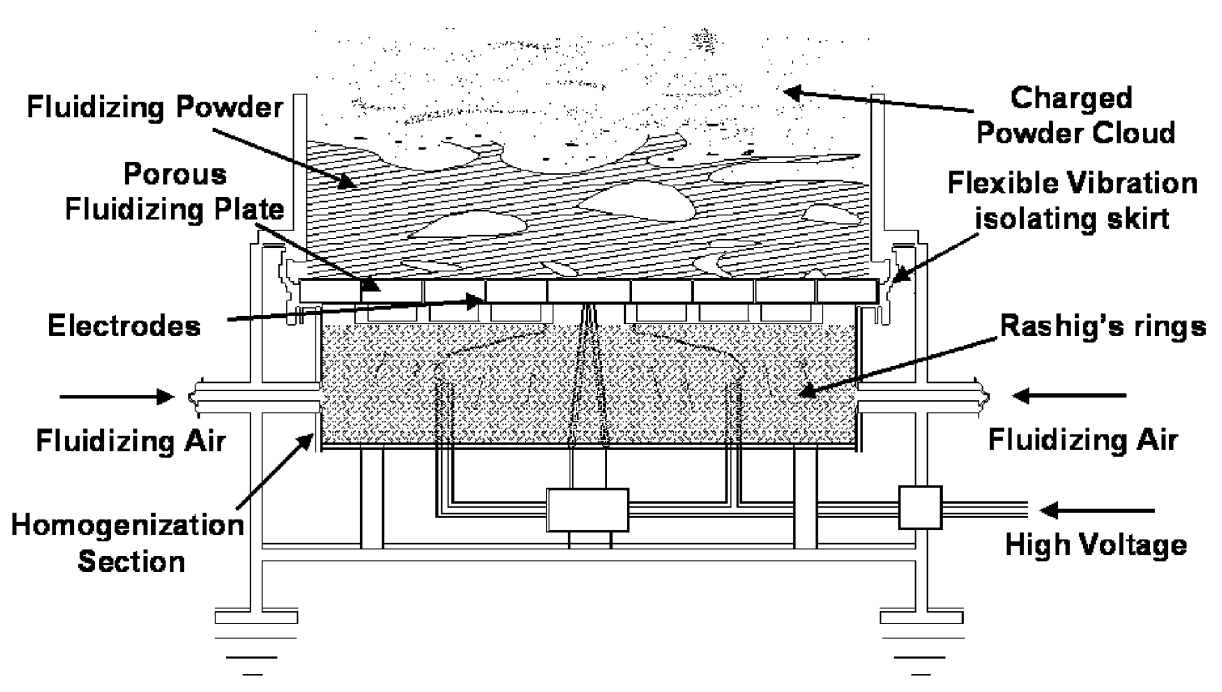

Fig. 1. EFB coating system.

grounded and, then, dipped in EFB in the zone immediately above the powder dense phase (Richardson, 1971) using a pneumatic movement system. Coating procedure was accurately described in a previous paper (Barletta and Tagliaferri, 2006b). However, it is important to remark that workpieces were generally placed at a distance of $100 \mathrm{~mm}$ away from the bed dense phase. The environmental conditions were always kept under firm control to minimize systematic error affecting the experimental procedure. Accordingly, all the tests were performed at a relative humidity below $40 \%$ with an average temperature of $20^{\circ} \mathrm{C}$ (accuracy $\pm 0.2^{\circ} \mathrm{C}$ ). Once the coating time was passed, the workpiece was removed from the column and, subsequently, located in a convective furnace Nabertherm, model B170 $30-2000^{\circ} \mathrm{C}$, to be cured at $170^{\circ} \mathrm{C}$ for about $10 \mathrm{~min}$. Afterward, the amount of deposited polymer onto workpiece surface was measured using a magnetic thickness gage (according to regulations ISO 2178 and ISO 2370). The coating thickness was estimated as the average of nine measurements equally spaced along the surface of each workpiece.

Two sets of experimental tests were defined following the DOE approach (Tables 1 and 2). First, a full factorial experimental plan, employing three factors (i.e. coating time, applied voltage and flow rate) and replicated four times for a total of 200 trials was performed. These tests were carried out to trace the experimental trends of coating thickness according to operational parameters as well as to examine their simple and interacted effects on EFB coating process. Second, a verification experimental plan (full factorial on the two factors 'coating time' and 'flow rate'), was performed in order to check process reproducibility and reliability by setting the electric parameters (applied voltage) at the level $(70 \mathrm{kV})$ found to be the best for the purposes of EFB coating process. A total of 80 experimental tests were carried out.
Table 1

Full factorial experimental plan

\begin{tabular}{llll}
\hline & \multicolumn{2}{l}{ Three factors } & \\
\cline { 2 - 4 } Levels & Exposure time $(\mathrm{s})$ & Airflow $\left(\mathrm{m}^{3} / \mathrm{h}\right)$ & Applied voltage $(\mathrm{kV})$ \\
\hline I & 7 & 3 & 50 \\
II & 14 & 5 & 60 \\
III & & 7 & 70 \\
IV & & 9 & 80 \\
V & & 11 & 90 \\
\hline
\end{tabular}

Table 2

Verification experimental plan

\begin{tabular}{lll}
\hline & \multicolumn{2}{l}{ Two factors } \\
\cline { 2 - 3 } Levels & Exposure time (s) & Airflow $\left(\mathrm{m}^{3} / \mathrm{h}\right)$ \\
\hline I & 4 & 3 \\
II & 7 & 5 \\
III & 11 & 7 \\
IV & 14 & 9 \\
V & & 11 \\
\hline
\end{tabular}

A strict statistical approach was followed in examining and reporting experimental data. All experimental results are reported as means with standard deviations to indicate their variability. The significance of operational parameters on the response coating thickness was found using ANOVA. A first approximation regression model was used to find the best fit for the experimental results as well as to provide a first check on the best of the developed neural network solutions. Finally, the experimental results of the verification experimental plan and a related, built ad hoc, physical model was used as the basis to assess the reliability of GA. 


\section{Neural network solutions}

\subsection{Neural networks models}

Three different neural networks were used to identify the most promising solution in the prediction of response, that is, the coating thickness in EFB coating process:

- RBF neural network,

- MLP neural network,

- GFF-MLP neural network.

Two different algorithms were used to train the MLP neural network:

- Gradient descent with momentum and adaptive learning rate $\mathrm{BP}$ algorithm,

- LM algorithm.

The algorithm of BP was the only used to train both the RBF and GFF-MLP neural networks.

The software simulator used was NeuroSolutions version 5.0 developed by NeuroDimension Incorporated.

\subsection{Radial basis function neural networks ( $R B F S$ )}

The radial basis function neural networks (RBFs) are non-linear hybrid networks (Haykin, 1994). They are typically constituted of a single hidden layer of processing elements (PEs), with this layer using Gaussian transfer functions. The centres and widths of the Gaussians are set by unsupervised learning rules and supervised learning is applied to the output layer. RBF networks are not affected by the problems of local minims. Besides, they demand shorter training time if compared with the MLPs. On the other hand, the scant properties of generalization represent the main drawback of RBFs.

\subsection{MLPs neural networks}

The MLPs are layered feed-forward neural networks (Haykin, 1994). The architecture of the MLPs is characterized by non-linear PEs, with the non-linearity function generally smoothed by logistic, sigmoid or hyperbolic tangent functions. Besides, the PEs are fully interconnected so that any element of the former layer feeds all the elements of the latter layer. The MLPs are easy to use and they can simulate any input/output map. Nevertheless, the training time of MLPs is very long and the training procedure requires many training data.

\subsection{Generalized feed-forward neural networks (GFF-MLP)}

The GFF-MLPs networks are a generalization of the MLPs in which connections between layers can jump over one or more layers (Principe et al., 2000). In theory, a MLP can solve any problem that a GFF-MLP network can solve. In practice, the GFF-MLPs often solve the problems much more efficiently. Besides, a standard MLP requires hundreds of times more training epochs than a GFF-MLP network constituted of the same number of PEs.

\subsection{Gradient descent with momentum and adaptive learning rate BP algorithm}

This method uses BP algorithm to calculate derivatives of performance cost function with respect to the weight and bias variables of the network (Haykin, 1994). Each variable is adjusted according to the gradient descent with momentum. For each step of the optimization, if performance decreases the learning rate is increased. When the BP algorithm is adopted, the operation of the neural network can be schematized into two main phases: forward computing and backward learning. In the forward phase, the synaptic weights are fixed, and the response of the network is computed by subjecting it to a prescribed set of input data. In the backward phase, the adjustments to synaptic weights are computed to minimize a cost function defined as the sum of error square.

\subsection{LM algorithm}

The LM algorithm is a second-order learning algorithm widely used in optimization problems (Principe et al., 2000). It outperforms simple gradient descent and other conjugate gradient methods in a wide variety of problems. The LM algorithm is generally adopted for the MLP network models as it trains significantly faster than momentum learning and usually it can converge with a lower error. On the other hand, the LM algorithm has great computational and memory requirements and thus it can only be used in small networks, even if more extensive applications can be found in the literature (Principe et al., 2000).

\subsection{Neural networks set-up}

The experimental factors having significant effects on the coating thickness, that is, coating time, voltage, and airflow were used as input PEs in all neural networks models. The coating thickness was the only output estimated, which, being defined as a normalized parameter, prevents the saturation of the chosen activation function.

A total of 50 samples coated with hybrid epoxy-polyester were analysed. As experimental data for simulation purposes, the averaged coating thicknesses of the four replications with each combination of experimental factors were used. Samples were shared according to the following percentages: $54 \%$ as training, $20 \%$ as cross-validation, and $26 \%$ as testing.

With regard to topological structure, just one hidden layer was chosen for all the network models. Moreover, a Gaussian transfer function was used only for RBF network model in both the hidden and output layers to generate the output values. In MLP and GFF-MLP networks, a sigmoid function was always used as a transfer function. 
As mentioned before, when BP algorithm was adopted as learning rule, it was based on the momentum with step size being set at 0.7 and a momentum coefficient for the hidden layer at 0.5 . The same operational parameters were used for the output layer, except for the step size, which was set at 0.1 . Besides, the rule of descendent gradient was adopted to calibrate the network and to identify the optimal weight values, that is, those minimizing the overall error between desired and calculated output. Instead, when the learning was performed by LM algorithm, only starting value of the parameter Lambda had to be set and a value of 0.01 was chosen. The Lambda parameter governs the step size, and it is dynamically adjusted according to error trends (Principe et al., 2000).

Afterward, all the chosen networks were thoroughly trained. Finally, in order to obtain a good indicator of generalization level achieved by the network, the network was stopped when the MSE of the cross-validation set began to increase, this being a signal that the network had come to the point of becoming over-trained.

Two different criteria are used to evaluate the effectiveness of each neural network model and its ability to make accurate predictions: the root mean square error (RMSE) and the correlation coefficient $(r)$. Therefore, the best fit between measured and estimated values, which is unlikely to occur, would have RMSE $=0$ and $r=1$.

\subsection{Genetic algorithm (GA)}

GA adopts Darwin's theory based on the emulation of the evolutionary process of the living organisms in which the principle of survival of the fittest rules (Goldberg, 1989). In recent years, GAs have found many applications in engineering sector and have been extensively used as a method of search in optimization problems (Goldberg, 1989). The main characteristic of parallel computing capability allows the combination of GAs with neural networks to have a higher expected probability to reach the best solutions. GAs employ a different learning strategy compared to neural networks. While neural networks have a learning strategy very close to living organisms during their life cycles, GAs employ a mechanism based on the selection of best samples to increase the probability that a whole class of organisms improves their performance.

Accordingly, in Fig. 2 the recursive strategy employed to determine the optimal architecture of neural networks using GA in the optimization problem of prediction of experimental trends of coating thickness in EFB process is reported.

\subsection{GA training options and basic genetic operators set-up}

The use of GA optimization requires the implementation of some basic tasks like the chromosome representation, the creation of the initial population, the evaluation function, the termination criteria, the selection function as well as the genetic operators for reproduction function, that is, crossover and mutation.

In this work, the chosen chromosomes were step sizes, momentum values and the number of PEs in the hidden layer. Then, to carry out the genetic training, a random

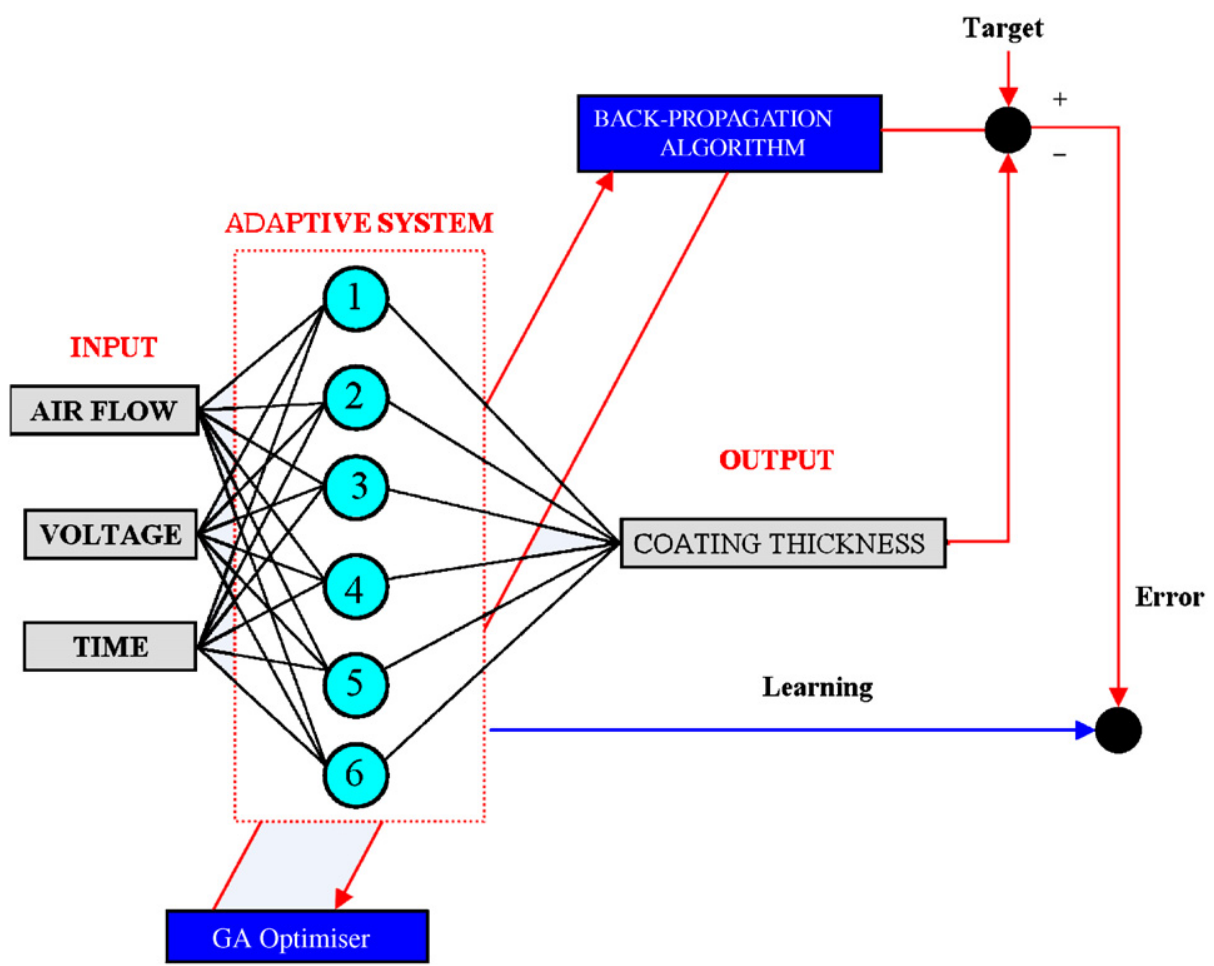

Fig. 2. The employment of GA in modelling of EFB coating process. 
initial population of 50 networks was generated. These networks were trained for 10000 epochs. Networks selection was led with the Roulette criteria in which the chance of a chromosome getting selected is proportional to its fitness or rank based on the minimum error achieved. The characteristics of the best networks were combined and mutated to create a new population of networks. One point crossover with 0.9 as crossover probability and uniform mutation operator with 0.01 as mutation probability was adopted as reproduction operators. The former is a crossover operator that randomly selects a crossover point within a chromosome and, then, interchanges the two parent chromosomes at this point to produce two new offspring. The latter is a mutation operator that replaces the value of the chosen gene with a uniform random value selected between the specified upper and lower bounds for that gene. The termination method was set-up on the generation number, fixed at 100 evolutions, that stops the evolution when the maximum number of generations was run.

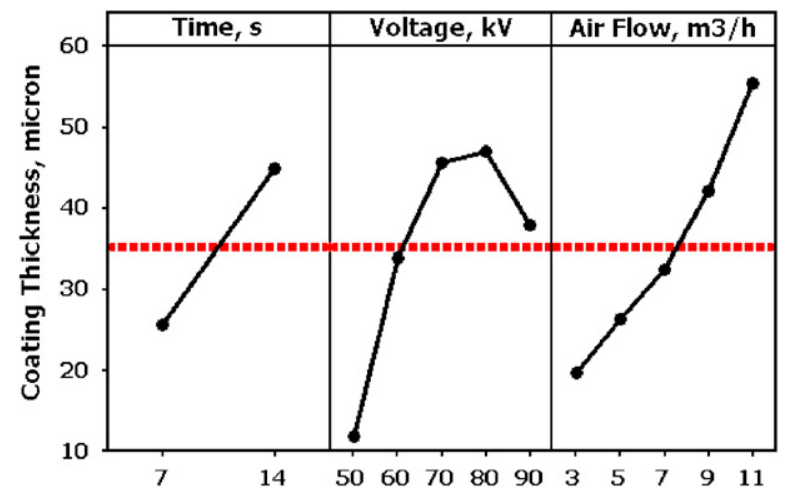

Fig. 3. Main effect plot.

\section{Simulation procedure and results}

\subsection{The experimental results and statistical approach}

The dimensional accuracy of any coating process has become a critical issue of any manufacturer because of increased quality demands. There are several factors that rules coating thickness in EFB deposition system and, hence, the development of an accurate experimentation as well as of analytical or empirical models for reliable prediction of coating thickness becomes a non-ignorable issue. Figs. 3 and 4 show, respectively, the analysis of mean (ANOM) and the interaction plot (IP) for the full factorial experimental investigation on the response coating thickness. Table 3 summarizes the results of the ANOVA on the same response.

The ANOM clearly demonstrates that with EFB system films less than $100 \mathrm{~mm}$ thick can be easily applied. Besides, even under the threshold of $100 \mathrm{~mm}$, EFB system was found able to manage the coating thickness accurately by

Table 3

Results of ANOVA table on full factorial experimental investigation considering interactions between factors

\begin{tabular}{lrrrrr}
\hline \multirow{2}{*}{$\begin{array}{l}\text { Interactions } \\
\text { Factors }\end{array}$} & DF & Seq SS & Adj MS & \multicolumn{1}{l}{$F$} & $\Pi(\%)$ \\
\cline { 2 - 6 } & 1 & 13945.6 & 13945.6 & 1233.46 & 18.96 \\
Time & 4 & 24074.8 & 6018.7 & 532.3 & 32.73 \\
Voltage & 4 & 23543.8 & 5886 & 520.6 & 32.01 \\
Airflow & 4 & 4205.2 & 1051.3 & 92.98 & 5.71 \\
Time*voltage & 4 & 2308.3 & 577.1 & 51.04 & 3.13 \\
Time*airflow & 16 & 3837 & 239.8 & 21.21 & 5.21 \\
Voltage*airflow & 16 & 502.8 & 31.4 & 2.78 & 0.68 \\
Time*voltage*airflow & 100 & 1130.6 & 11.3 & & 1.53 \\
Error & 149 & 73548.1 & & & \\
Total & & & & &
\end{tabular}

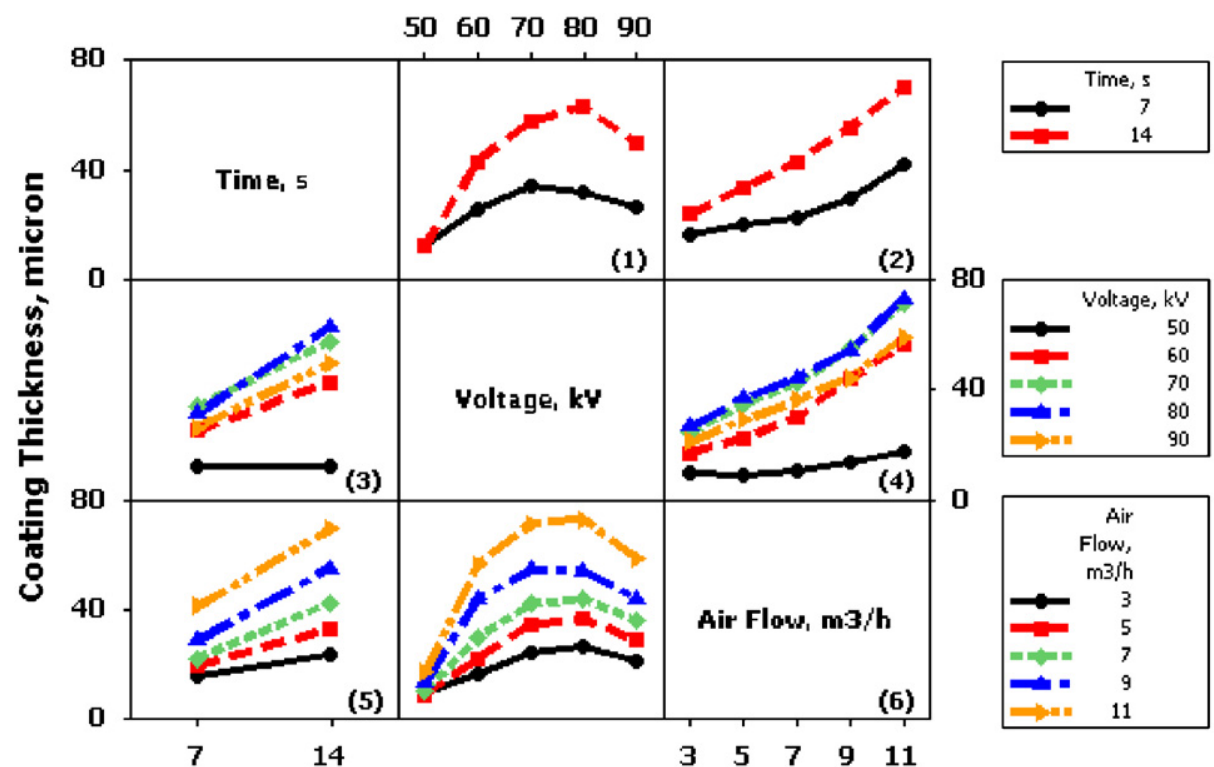

Fig. 4. Interaction plot. 
just regulating the most appropriate levels of the experimental factors coating time, applied voltage and flow rate (Fig. 3).

Watching the panels 1 and 2 shown in the first row of Fig. 4, a similar trend seems to characterize the coating thickness for both the exposure times (7 and 14s) and varying all the applied voltages and flow rate. These results comply with the trends reported in the literature (Barletta and Tagliaferri, 2006a; Barletta and Tagliaferri, 2006b; Barletta et al., 2005). Of relevance, an initial rise in coating thickness is obtained from increasing the applied voltage. Then, after a maximum value has been achieved, a strong decrease in thickness clearly occurs in agreement with data reported in the literature (Barletta and Tagliaferri, 2006a; Barletta and Tagliaferri, 2006b; Barletta et al., 2005). These highly non-linear trends of the coating thickness according to operational variables are ascribable to the strong back ionization phenomena (Guskov, 2002; Barletta and Tagliaferri, 2006a; Barletta and Tagliaferri, 2006b; Barletta et al., 2005), which characterize all the electrostatic deposition techniques and make all the modelling and simulation attempts very troubled. Besides, this trend is common to all the investigated airflow and coating time levels, even if a top shift of all the curves is clearly evident (panels 1 and 6 in Fig. 4), hence making all predictive procedures even more complicated.

ANOVA has been carried out to find the effect of experimental factors on the response coating thickness. Since the experiments were led using a full factorial design and four replications of experiments with each combination were carried out, the three factors interaction was also considered. Examination of the data collected in Table 3 reveals the significance of all the simple experimental factors 'applied voltage', 'airflow' and 'coating time' on achievable coating thickness in EFB coating process. However, slightly lower values of Fisher's factor and of contribution percentage $P(\%)$ were found for the experimental factor 'time' (Table 3). Besides, even the interactions among investigated experimental factors had a lower influence on the values of response. At best, their contribution percentage does not overcome the value of $5 \%$. Lastly, by disregarding all the interactions, a contribution percentage $P(\%)$ for residuals of about $15 \%$ with an overall $\mathrm{MS}_{\mathrm{w}}$ of 85.6 confirms the low incidence of the systematic errors on reliability of collected data and of the whole experimental procedure. It should be borne in mind that EFB coating process, as all the other coating processes, typically posses a natural variability of results due to unpredictable factors very high (Barletta and Tagliaferri, 2006b).

\subsection{Mathematical formulation}

Since all experimental factors were found to be influent and the interactions among them could be disregarded without affecting too much the overall error (low values of the contribution percentages), only the simple factors 'applied voltage', 'airflow' and ' coating time' were considered in the regression model. Consequently, a multiple regression analysis was led to find the best mathematical model for EFB coating process with the parameters (coating time $t$, applied voltage $V$ and air flow $F$ ) under consideration.

The model can be so formulated:

$c=f(t, V, F)$,

where $c$ is the coating thickness, $f$ the response function, and $t, V$ and $F$ the EFB variables. Expressed in non-linear form Eq. (1) becomes:

$c=C t^{\alpha} V^{\beta} F^{\gamma}$.

To simplify the determination of the constant and parameters, the mathematical model was linearized performing a logarithm transformation. Eq. (2) becomes:

$\ln c=\ln C+\alpha \ln t+\beta \ln V+\gamma \ln F$.

The constant $C$ and parameters $\alpha, \beta, \gamma$ were solved by using a multiple regression analysis with the assistance of experimental results. Eq. (3) can be so rearranged as:

$c=6.49 \times 10^{-4} t^{0.673} V^{1,85} F^{0,721}$.

A value of $R^{2}$ higher than $72 \%$ was found and the results of analysis of residuals are shown in Fig. 5. Residuals distribute not so far from a normal distribution. No evident bias or peculiar patterns affect residual distribution with respect to fitted values.

The major finding of the regression model is the nonlinear relationship between applied voltage and coating thickness in agreement with previous experimental indications and the literature (Barletta and Tagliaferri, 2006b). This provides an opportunity to maximize the coating thickness by selecting the proper coating process parameters, that is, highest values of coating time and airflow and intermediate value for applied voltage (as previously seen, not higher than $70 \mathrm{kV}$ ). In this way, both the fastest and the best overall performance of EFB coating process could be achieved in agreement with the data reported in the literature (Barletta and Tagliaferri, 2006a; Barletta and Tagliaferri, 2006b; Barletta et al., 2005), as the back ionization phenomena would be minimized with concurrent advantages of both functional and aesthetic aspect of the applied coating.

\subsection{The results of the verification experimental plan and the analytical model}

The trends of coating thickness according to coating time with flow rate for verification experimental plan is shown in Fig. 6. The verification experimental plan was led setting the applied voltage at $70 \mathrm{kV}$. In such a way, as indicated by experimental results reported in the previous section, the fastest coating process and the best coating condition can be expected. To fit the experimental data, a mono-parametric analytical model, which relates coating 

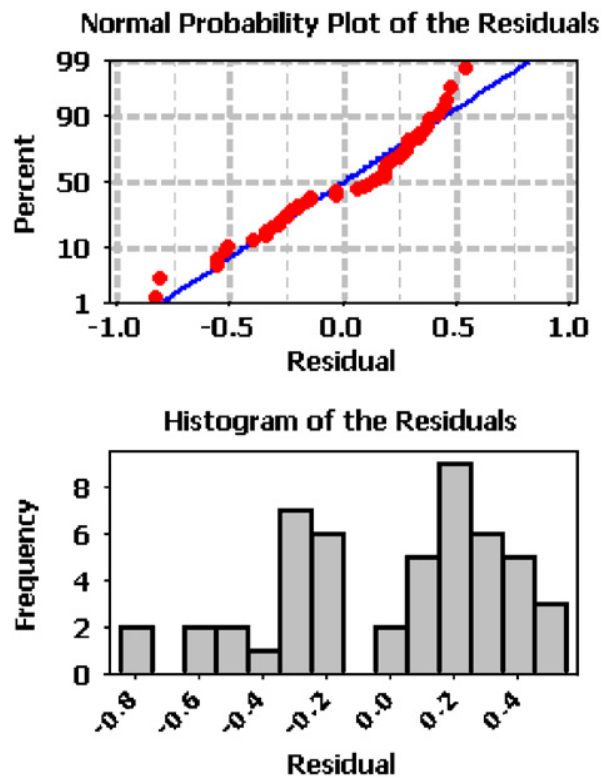

Residuals Versus the Fitted Values

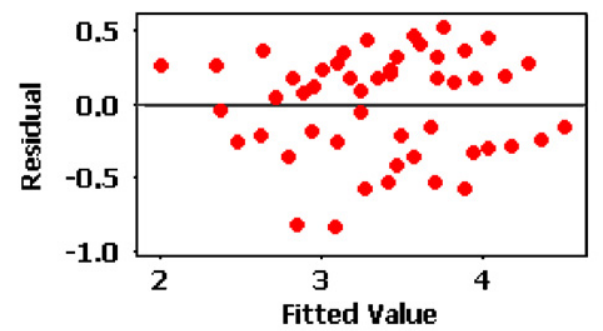

Residuals Versus the Order of the Data

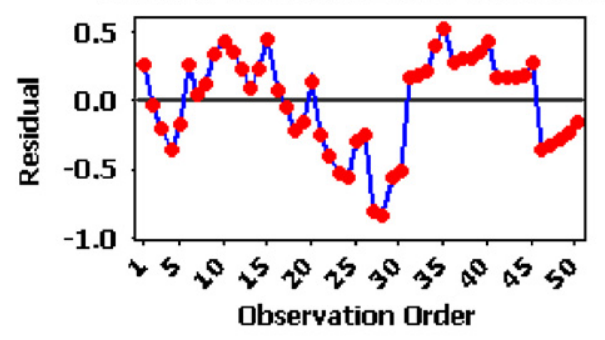

Fig. 5. Analysis of residuals for regression model.

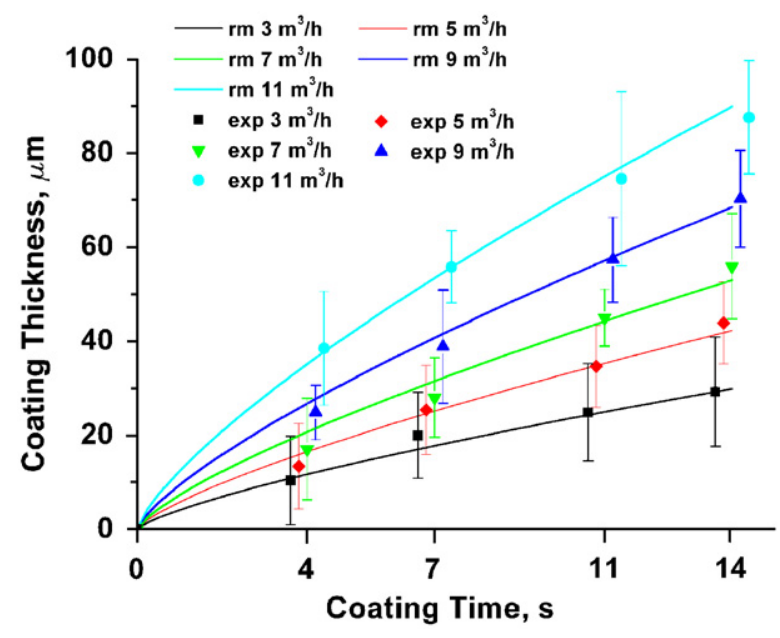

Fig. 6. Validation of regression model: comparison with experimental data.

thickness and coating time, was calculated and assessed to be accurate for each airflow investigated:

$c=K t^{0.75}$,

where $c$ is the coating thickness, $t$ the coating time and $K$ an empirical constant. A physical interpretation of constant $K$ can be provided. $K$ is a strict linear function of airflow. In fact, when its value for each curve shown in Fig. 6 at different airflows is calculated, the resulting trend is linear. Besides, $K$ is worth 0 if airflow is set at $0.65 \mathrm{~m}^{3} / \mathrm{h}$, that is, the airflow corresponding to the minimum fluidization. In the light of this, Eq. (5) can be rearranged:

$s=\left(F_{\mathrm{s}}-F_{\mathrm{m}}\right)\left(t-t_{0}\right)^{p}$,

where $F_{\mathrm{s}}$ is the current airflow value and $F_{\mathrm{m}}$ the minimum fluidization airflow. A correlation coefficient $r$ of approximately 0.95 and a $p$-value of more than 0.1 confirm the accuracy of both the analytical model and the calibration constant.

\subsection{Neural network approach}

A set of different neural network models have been examined to find the best solutions in predicting the experimental trends of coating thickness in EFB coating process with respect to all the investigated operational parameters. Fig. 7 displays the performance of the four neural networks employed. The effect of RMSE on the number of PEs in the hidden layer is reported. RMSE is minimized when the number of PEs is set at 3 for both GFF-MLP and MLP neural networks with LM algorithm. Instead, RMSE is minimized when five PEs for RBF and six PEs for MLP neural network with BP algorithm are used.

The best PEs values were adopted in the hidden layer to find the best topology of the neural networks. First, the epochs number for each neural network model was fixed to 1000, except for RBF model in which 1100 epochs, 1000 for supervised learning and 100 for unsupervised learning, were employed. Fig. 8 shows the RMSE trends of all the chosen models according to the number of epochs. The RBF topology was discarded as it exhibited performances worse than MLP topology. In fact, data collected in Table 1 show that MLP and GFF-MLP topologies assure a better learning as the calculated errors are significantly lower than for RBF model. Nevertheless, the detected RMSE for all the MLPs models being very close, a further training with 10000 epochs was carried out to find the best model. The training was repeated five times to minimize the results variability and the network weights, which minimized the RMSE of the cross-validation, were chosen as the best weights (Table 4). 

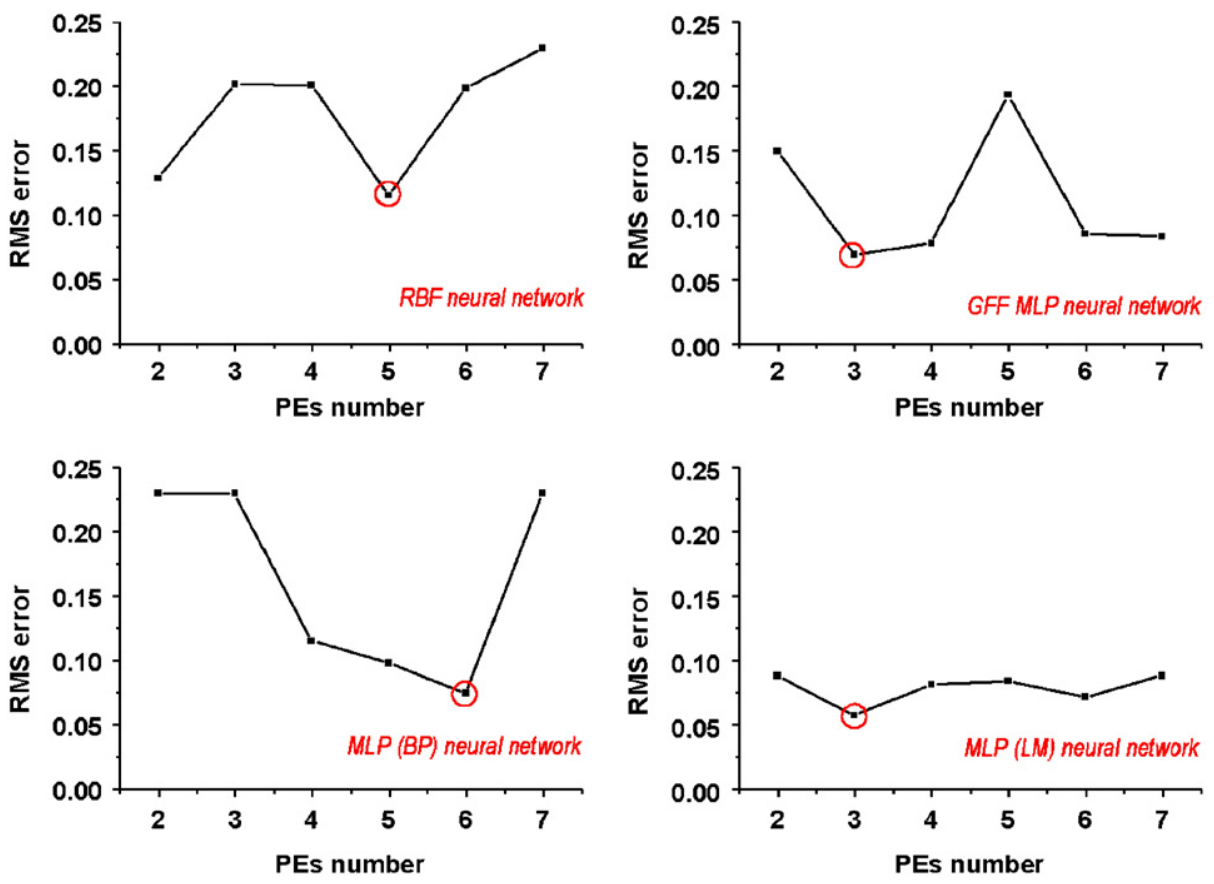

Fig. 7. Analysis of the best PEs number in the hidden layer for the different neural network models.

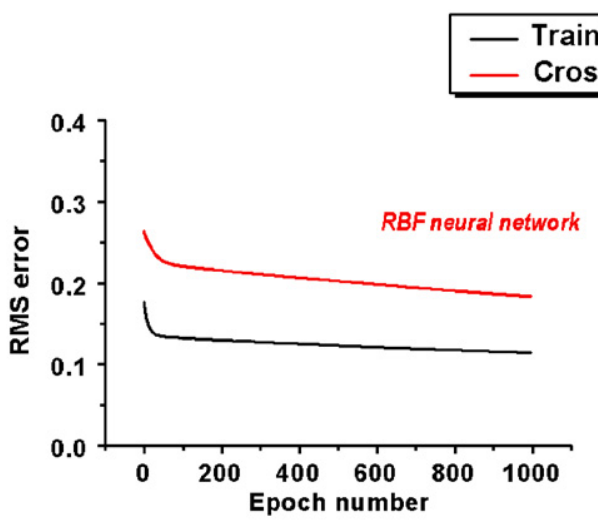

Training Set
Cross Validation Set
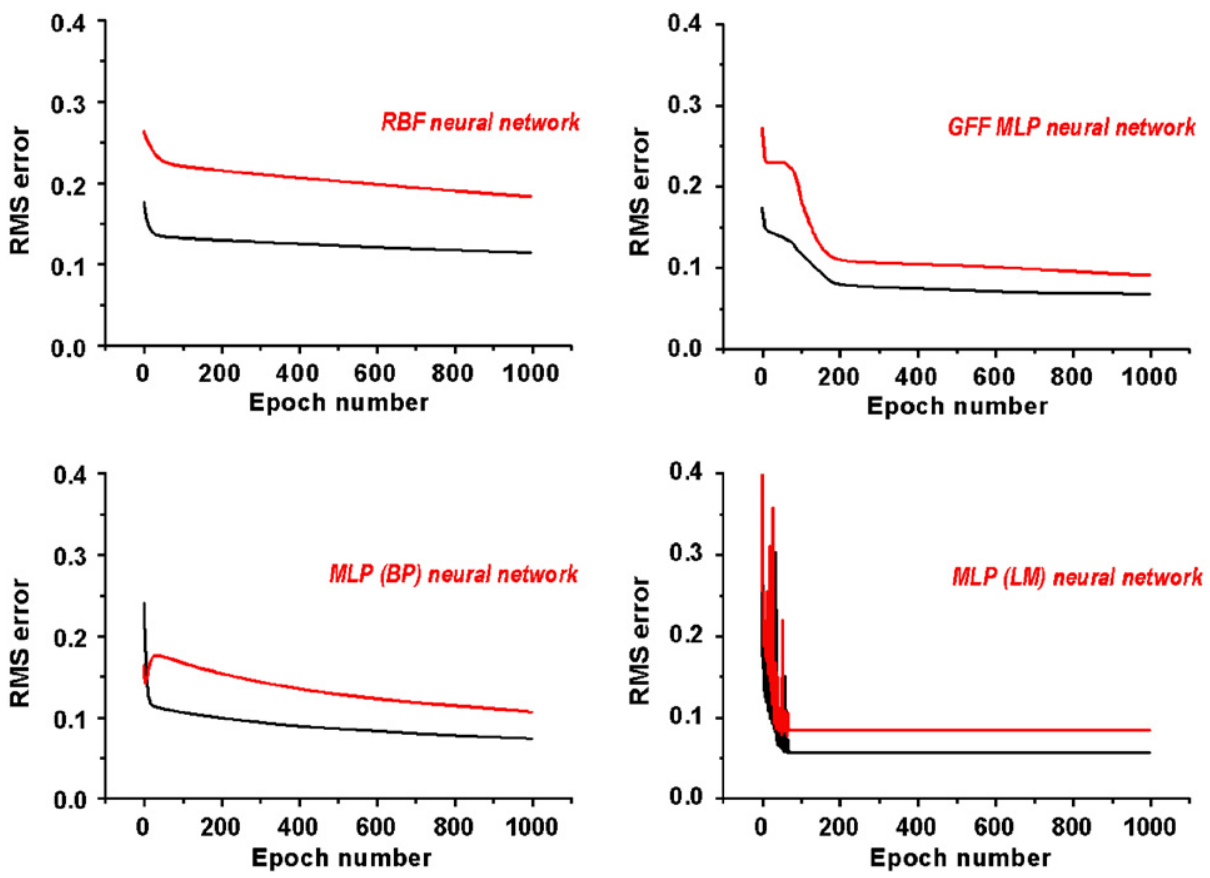

Fig. 8. Analysis of the best topology for the different neural network models over 1000 epochs.

The trends of RMSE for training and cross-validation set during the learning process with the best weights of all the MLP models are shown in Fig. 9. All the employed MLP models allowed achieving very low values of RMSE (Table 5). Nevertheless, the lowest RMSE value was achieved using the MLP model with BP algorithm. In fact, increasing the number of epochs, such a network model gave the best overall performance, hence reducing the error of about $30 \%$ for training set and more than $50 \%$ for cross-validation set. This behaviour is in agreement with theoretical indications (Haykin, 1994; Principe et al., 2000). In fact, MLP with LM algorithm and GFF-MLP models 
were faster to converge, but MLP with BP algorithm, even if slower to converge, allowed achieving best results on long training time.

\subsection{Modelling of experimental data}

In the light of previous results, the modelling of experimental data for EFB coating process was performed using the MLP model with BP algorithm as it guarantees the best simulative performance with the lowest errors. The capabilities of this MLP model were checked by comparison with experimental data coming from full factorial experimental plan and the built ad hoc regression model. Fig. 10 shows the comparison between the output calculated by the network and the desired output, that is, the experimental one. Besides, the errors of MLP model in fitting the experimental results are provided in Table 6 and a comparison with the regression model was also performed. As can be seen, the correlation coefficient $r$ is very close to 1 for training (0.975) and cross-validation set

Table 4

Best RMSE for the different neural network models over 1000 epochs

\begin{tabular}{lll}
\hline Networks & RMSE training & RMSE cross validation \\
\hline RBF & 0.11571 & 0.18494 \\
GFF MLP & 0.06991 & 0.09276 \\
MLP BP & 0.05730 & 0.10828 \\
MLP LM & 0.05809 & 0.08491
\end{tabular}
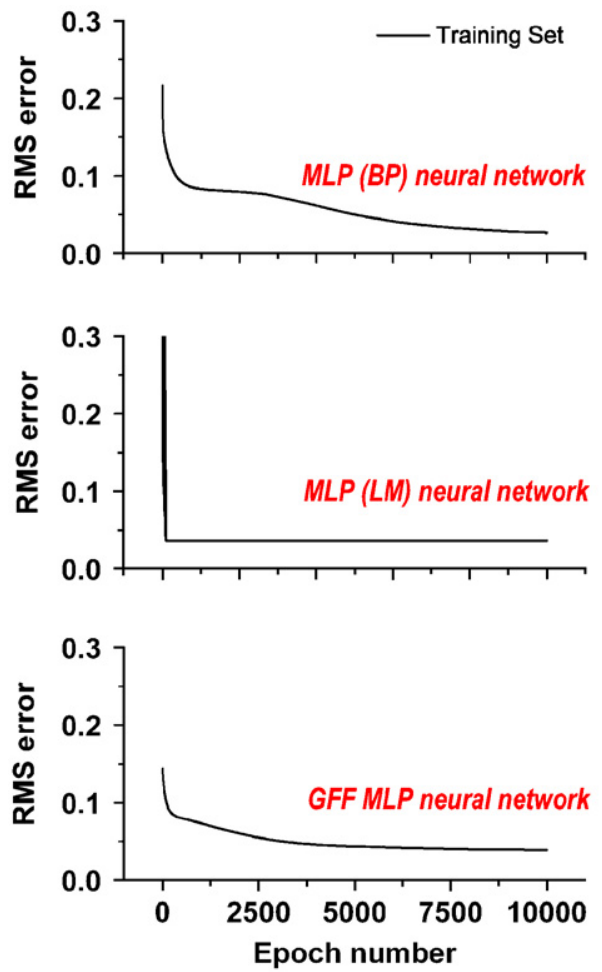

(0.988). Instead, it assumes a slightly lower value for testing set (0.928), even if it keeps guaranteeing a very good fitting. Besides, very low standard errors (less than $5 \%$ ) were calculated for both training and cross-validation set. Testing set exhibited a slightly larger standard error (close to $8 \%$ ). However, MLP model is affected by standard error definitely lower than the regression model. In fact, standard errors in the range of $12-16 \%$ and correlation factors in the range of $0.80-0.83$ were detected for regression model. In addition, the error of $8 \%$ for testing set of MLP model with BP algorithm is definitely noteworthy. In fact, as the results of ANOVA highlight and data reported in the scientific and technical literature confirm (Guskov, 2002; Barletta and Tagliaferri, 2006a; Barletta and Tagliaferri, 2006b; Barletta et al., 2005), the variability of coating process due to systematic error and to the unpredictable factors of the coating procedure is quite higher. It is worth about $15 \%$ even if, as in our experimentations, all cautions to ensure the best accuracy of coating tests are taken. Finally, mean error larger than

Table 5

Best RMSE for the different neural network models over 10000 epochs

\begin{tabular}{lll}
\hline Networks & RMSE training & RMSE cross validation \\
\hline GFF MLP & 0.03887 & 0.07321 \\
MLP BP & 0.02682 & 0.03435 \\
MLP LM & 0.03629 & 0.06707 \\
\hline
\end{tabular}
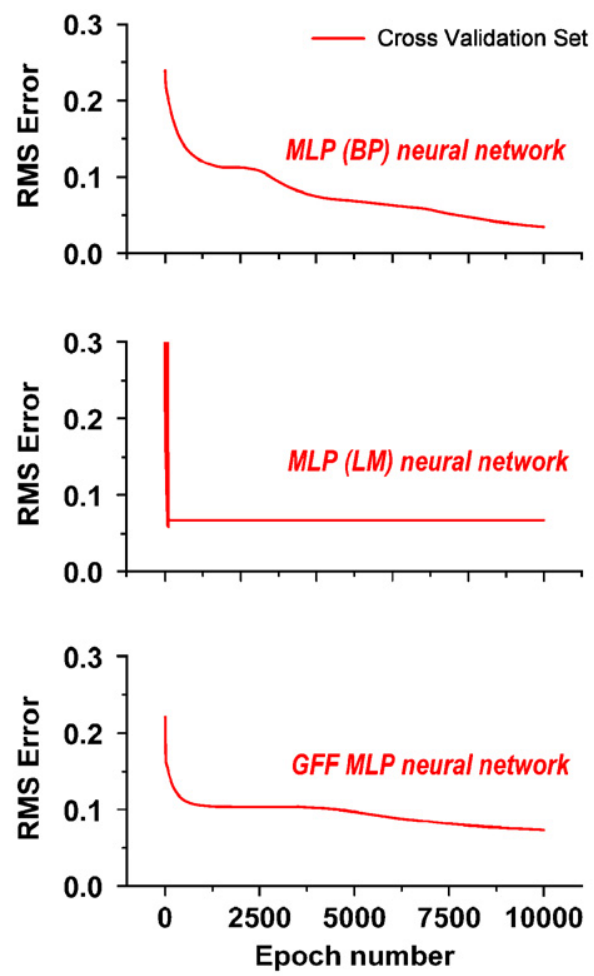

Fig. 9. Analysis of the best topology for the different neural network models over 10000 epochs. 

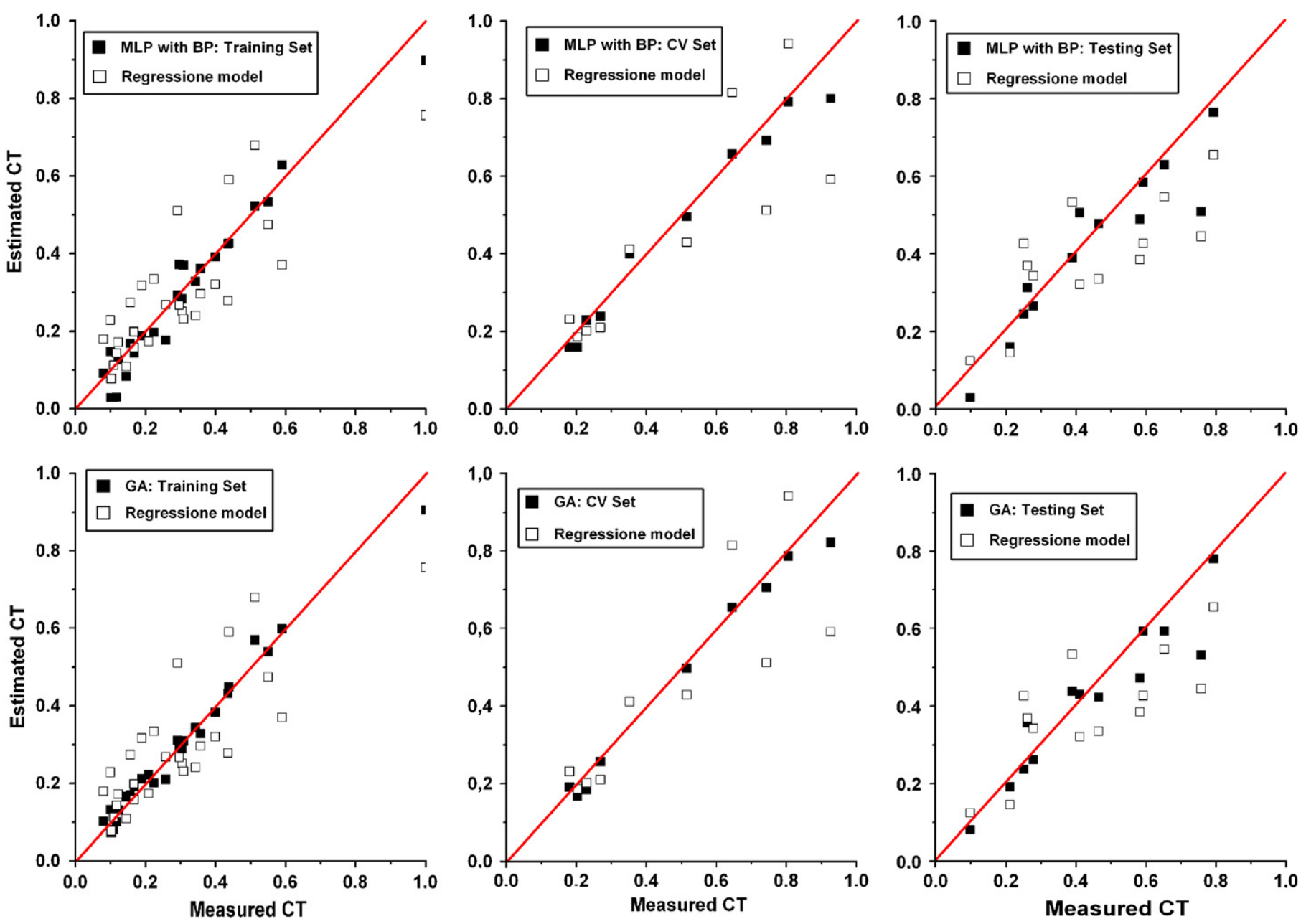

Fig. 10. Check of MLP model with BP algorithm and with GA on experimental data and regression model for training, cross-validation and testing set.

Table 6

Correlation factor and standard error: MLP model with BP algorithm and regression model

\begin{tabular}{llll}
\hline Correlation & Training set & CV set & Testing set \\
\hline MLP with BP & 0.97493752 & 0.987766163 & 0.928084951 \\
GA & 0.990311622 & 0.989131454 & 0.938654488 \\
Regression & 0.824944364 & 0.83608228 & 0.806537038 \\
\hline
\end{tabular}

$8 \%$ are quite common in all the applications of neural network models in predicting data concerning machining operations (Jain and Jain, 2000; Gopal and Rao, 2003), fault detection of mechanical components (Letter to the Editor, 2004) and in several laser processing of metal components (Jenga et al., 2000; Koa et al., 1999; Cheng and Lin, 2000), hence confirming the reliability of the developed MLP model with BP algorithm and its suitability for the analysis of EFB coating process.

Fig. 10 displays also the fitting between the results of the MLP model with GA and the experimental data. A comparison with the regression model is also reported.
Table 7

Correlation factor and standard error: MLP model with GA and regression model

\begin{tabular}{llll}
\hline Error & Training set & CV set & Testing set \\
\hline MLP with BP & 0.046052688 & 0.045884538 & 0.085630417 \\
GA & 0.028744341 & 0.04326332 & 0.079304292 \\
Regression & 0.11699819 & 0.161421509 & 0.151235368
\end{tabular}

The optimization results of the MLP model with BP algorithm match very well with the results achieved using GA. However, a further improvement of fitting can be underlined as a result of the application of GA. In particular, the correlation coefficient $r$ moves from 0.928 for testing set using the MLP model with BP algorithm to 0.939 for testing set using the GA (Table 7). A certain improvement regards also the standard errors. In fact, standard errors of both the training $(2.9 \%)$ and crossvalidation $(4.3 \%)$ set were always found to be quite lower for GA. Moreover, an improvement of more than $0.6 \%$ was found for standard error of testing set using GA. 


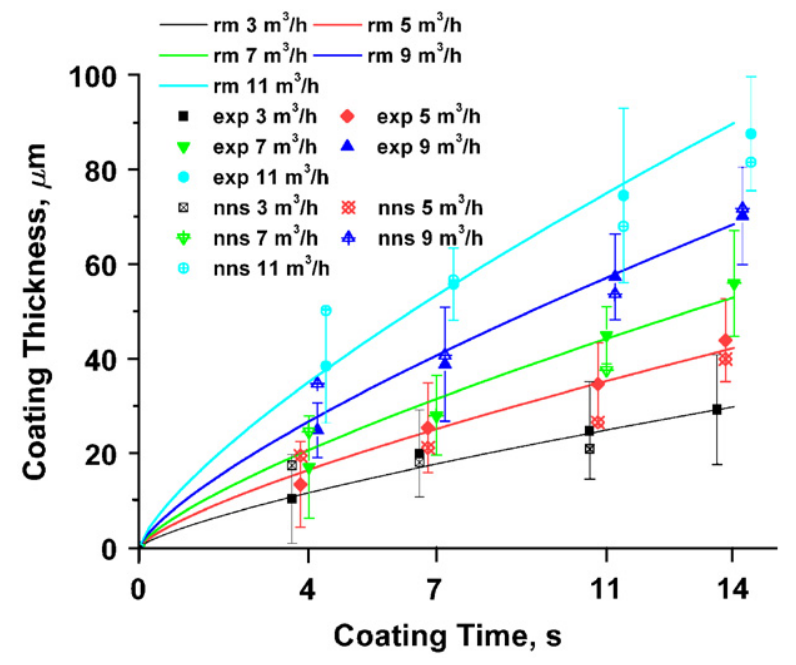

Fig. 11. Performance of the MLP model with GA in predicting a verification set of experimental data and comparison with the analytical model.

\subsection{Optimization using GAs}

In the light of previous results, the MLP model with GA was used to predict the results of the verification experimental plan. The neural network model was also tested for $4 \mathrm{~s}$ as coating time, that is, outside of the range, which was calibrated for. A comparison with the analytical model was also performed. As Fig. 11 shows, an appreciable fitting between calculated and desired output was found, with no significant deviations or anomalies arising. The output provided by the neural network model with GA is well inside the error bar representing the variability of results of the experimental tests. However, if used between the prescribed ranges, that is, for coating time in the range of $7-14 \mathrm{~s}$, the standard error of MLP model with GA is worth about $3.9 \%$ with an outstanding correlation factor of 0.986 . As comparison, the analytical model produces an error around $2.4 \%$ in fitting the experimental data with a correlation factor of 0.995 . If MLP network with GA is also used to predict data outside the range, which was designed for, the error grows up to $6.5 \%$ with a correlation factor of 0.963 . Even if this value can be considered rather high, it is a quite good value because of the scant capacity of neural network to extrapolate results outside the prescribed ranges (Principe et al., 2000). Besides, such a value of mean error is well below the natural variability of EFB coating process that, as seen, is worth around $15 \%$. On the other hand, the performance improvement ascribable to the employment of GA is characterized by a quite higher computational time.

All these findings confirm how good and reliable the MLP model with GA is in predicting the experimental trends of coating thickness according to all the operational parameters of EFB coating process.

\section{Conclusions}

In this paper, the effectiveness of using different neural network solutions for modelling and prediction of coating thickness trends in EFB coating process is comparatively evaluated. MLP model with BP algorithm was found to be the best neural network solution. For such neural network model, simulation results display a very good agreement with available experimental data for a wide range of operational parameters of EFB coating process.

The employment of GA improves the capability of the developed neural network to predict the experimental results. A further improvement of the fitting between the simulation and the experimental data was found. Besides, the optimization results of the neural network with and without GA match very well, thus confirming the reliability of the neural network approach.

A check of neural network solution with GA was also performed on a defined ad hoc verification experimental plan. The comparison stated that fair results could be even achieved in predicting experimental data outside the range, which the neural network was designed for, indicating also a certain capability of the neural network model with GA to extrapolate. Finally, a comparison with a regression model built on data of verification plan stated, once more, the reliability of neural network with GA to match the experimental data and as valid and universal alternative to analytical models.

Finally, the developed neural network system with GA is a wide-spectrum instrument and can be easily extended to other coating processes to improve the overall efficiency and to simplify the operative choices. In fact, the discussed system is fairly general and its optimization does not involve process models or constraints, but only purely experimental observations. Therefore, this neural network solution assisted by GA forms the basis for the development of a fast prediction model to estimate the effectiveness of the EFB coating process accurately, hence becoming a support tool for the purposes of process automation and control.

\section{References}

Ali, F.S., Inculet, I.I., 2000. IEEE Transactions on Industry Applications 36 (5).

Anon, A., 1995. Industrial Paint and Powder 10, 8.

ASM Metal Handbook, 1995. Surface Cleaning, Finishing, and Coating, ninth ed.

Barletta, M., Tagliaferri, V., Santo, L., 2005. International Journal of Production Research, submitted for publication.

Barletta, M., Tagliaferri, V., 2006a. Surface and Coatings Technology. 200 (14-15), 4282-4290.

Barletta, M., Tagliaferri, V., 2006b. Surface and Coatings Technology. 200 (14-15), 4619-4629.

Barletta, M., Simone, G., Tagliaferri, V., 2006. International Journal of Material Processing, in press.

Barletta, M., Simone, G., Tagliaferri, V., 2005. Progress in Organic Coatings. 54 (4), 390-398. 
Cheng, P.J., Lin, S.C., 2000. International Journal of Machine Tools \& Manufacture 40, 1185-1197.

Cho, H.S., 2001. Neural Network Applications to Manufacturing Processes: Monitoring and Control, Korea Advanced Institute of Science and Technology (KAIST). CRC Press LLC.

Cudin, C., 2005. Internal Report, Electrolux Group, Porcia.

Goldberg, D.E., 1989. Genetic Algorithm in search, Optimization \& Machine Learning. Addison-Wesley Publishing Company.

Gopal, A.V., Rao, P.V., 2003. International Journal of Machine Tools \& Manufacture 43, 1237-1336.

Guskov, S., 2002. Electrostatic Phenomena in Powder Coating. Powder System Group Nordson Corporation.

Haykin, S., 1994. Neural Network: A Comprehensive Foundation. Macmillan, New York.

Jain, R.K., Jain, V.K., 2000. Journal of Materials Processing Technology $108,62-67$.
Jenga, J.-Y., Maub, T.-F., Leuc, S.-M., 2000. Journal of Materials Processing Technology 99, 207-218.

Koa, D.-C., Kimb, D.-H., Kimc, B.-M., 1999. International Journal of Machine Tools \& Manufacture 39, 771-785.

Letter to the Editor, 2004. Mechanical Systems and Signal Processing. 18, $1273-1282$

Principe, J.C., Euliano, N.R., Lefebvre, W.C., 2000. Neural and Adaptive Systems: Fundamentals Through Simulations. Wiley.

Richardson, J.F., 1971. Incipient fluidization and particulate systems. In: Davidson, J.F., Harrison, D. (Eds.), Fluidization. Academic Press, New York.

Weiss, K.D., 1997. Progress in Polymer Science 22, 203-245.

Ye, Q., Domnick, J., 2003. Powder Technology 135-136, 250-260.

Ye, Q., Steigleder, T., Scheibe, A., Domnick, J., 2002. Journal of Electrostatics 54, 189-205. 